\title{
Alfapeguinterferon-2a e ribavirina versus alfapeguinterferon-2b e ribavirina: avaliação custo-efetividade e do impacto orçamentário do tratamento do genótipo 1 da hepatite C crônica
}

I ${ }^{1}$ Gabriela Bittencourt Gonzalez Mosegui, ${ }^{2}$ Cid Manso de Mello Vianna,

${ }^{3}$ Marcus Paulo da Silva Rodrigues, ${ }^{4}$ Renata de Mello Perez I

Resumo: Foi realizada análise de custo-efetividade e do impacto orçamentário de tratamentos indicados para adultos infectados com genótipo 1 do vírus da hepatite $\mathrm{C}$, comparando o não-tratamento com terapias combinadas de alfapeguinterferon-2a e $2 \mathrm{~b}$ associados a ribavirina. $\mathrm{O}$ modelo de Markov desenvolvido projetou a evolução da hepatite C em coorte de 1.000 pacientes, por um período de 30 anos, para os vários estados do desenvolvimento da doença. As terapias combinadas da ribavirina com o alfapeguinterferon $2 \mathrm{a}$ ou $2 \mathrm{~b}$ apresentam efetividades estatisticamente idênticas quando avaliadas em 30 anos de evolução da doença. Quanto ao impacto do tratamento nos anos de vida e nos anos de vida ajustados por qualidade de vida; os anos de vida ganhos por qualidade de vida em relação à evolução da doença sem tratamento foram de 1,67 e 1,63, respectivamente, para a terapia combinada com alfapeguinterferon $2 \mathrm{a} e \mathrm{~b}$, aplicando-se 5\% de desconto. A estratégia de tratamento com alfapeguinterferon $2 \mathrm{a}$ mais ribavirina se revelou mais custo-efetiva, dominando a outra alternativa de tratamento. Embora não haja diferenças significativas de efetividade entre os dois tipos de alfapeguinterferon, a diferença de preço entre os dois medicamentos faz com que a alternativa do uso do alfapeguinterferon 2a mais ribavirina seja mais eficiente. Quanto à estimativa do impacto orçamentário para o período de 2008 a 2017, a utilização do alfapeguinterferon 2a mais ribavirina resulta em redução nos gastos de aproximadamente $19 \%$, caso todos os doentes fossem tratados utilizando-se os esquemas terapêuticos selecionados.

> Palavras-chave: avaliação de custo-efetividade, hepatite C crônica, genótipo 1, interferons peguilados, ribavirina.
1 Professora adjunta no Instituto de Saúde da Comunidade, Universidade Federal Fluminense. Endereço eletrônico: gabrielamosegui@ uol.com.br

${ }^{2}$ Professor adjunto no Instituto de Medicina Social, Universidade do Estado do Rio de Janeiro (IMS-UERJ). Endereço eletrônico: cmmv@ ims.uerj.br

${ }^{3}$ Especialista em Farmácia Industrial; pesquisador associado no IMS-UERJ Endereço eletrônico: mps rodrigues@yahoo.com.br

${ }^{4}$ Professora adjunta no Departamento de Clínica Médica, Faculdade de Medicina da Universidade Federal do Rio de Janeiro; doutora em Gastroenterologia. Endereço eletrônico: renatamperez@ gmail.com 
A infecção pelo vírus da hepatite C (VHC) afeta tanto países desenvolvidos quanto aqueles em desenvolvimento, sendo reconhecido como importante problema de saúde pública, tendo em vista o aumento da detecção de novos casos, a gravidade das complicações derivadas da infecção crônica e seu impacto econômico para a sociedade. $\mathrm{O}$ número de infectados, segundo a Organização Mundial da Saúde, pode chegar a cerca de 3\% (170 milhões) da população mundial (WHO, 2000). Quanto às estimativas de mortalidade por causas relacionadas à hepatite viral crônica C (HVC), há uma notável abrangência na contagem dos casos, podendo variar entre um milhão a aproximadamente 14 milhões de mortes em todo o mundo (MURRAY; LOPEZ, 1997; LAVANCHY, 2002). No Brasil, ainda é desconhecido o número real de infectados, e ainda segundo as variaçōes consideráveis nas estimativas de 1.6\% a 4,9\%, acredita-se que não se esteja nem próximo da atual realidade, entre outros fatores, devido à subnotificação de casos (FONSECA, 1999; WHO, 2000).

$\mathrm{O}$ início do tratamento para erradicação do vírus da hepatite $\mathrm{C}$ está indicado para os pacientes portadores da infecção crônica, quando estes possuem certas características. Os tratamentos farmacológicos disponíveis são: interferon alfa-2a ou alfa- $2 \mathrm{~b}$; interferon peguilado alfa- $2 \mathrm{a}$ ou alfa- $2 \mathrm{~b}$ e ribavirina. $\mathrm{O}$ uso do interferon convencional ou interferon peguilado é dependente, dentre outros fatores, do genótipo viral, devido a sua influência na agressividade da infecção e da resistência ao tratamento farmacológico (BRASIL, 2007; HNATYSZYN, 2005).

Quanto à relação custo-efetividade dos interferons peguilados, uma análise da literatura internacional mostra resultados contraditórios. Segundo Malone et al. (2005), a utilização de alfapeguinterferon $2 \mathrm{~b}$ se revela mais eficiente pela maior probabilidade do valor preditivo da resposta viral sustentada (RVS). O estudo, no entanto, baseia-se em uma árvore de decisão, sem considerar a evolução da doença, nem utilizar como medida de efetividade a qualidade de vida dos doentes. Já Yeh et al. (2007), utilizando um modelo de Markov para a evolução da história natural da hepatite $\mathrm{C}$ e medidas de utilidade para avaliar o desfecho da evolução da doença, não encontraram resultado que desse preferência a um ou outro tipo interferon peguilado. Ainda segundo o estudo, para o genótipo tipo 1, não existiriam recomendações privilegiando o uso de um ou outro medicamento. 
No Brasil, o Ministério da Saúde (MS) distribui, desde 1999, combinações desses medicamentos através das Secretarias Estaduais de Saúde (SES), envolvendo um significativo consumo de recursos para o Sistema Único de Saúde (SUS). Em 2000, foi publicado o primeiro protocolo nacional traçando as diretrizes para o tratamento da hepatite C crônica no Brasil (BRASIL, 2000). Atualmente, a portaria SVS/MS no 34/2007 é a norma legal que rege o protocolo clínico e as diretrizes terapêuticas para o tratamento da hepatite viral crônica C. O processo de aquisição do alfapeguinterferon é norteado pela Portaria no 3.227/ 2006, que centraliza na esfera federal a compra do medicamento, assim como a ribavirina, que é assegurada da mesma forma, pela Portaria no 1.259/ 2009 (BRASIL, 2009a; BRASIL, 2007; BRASIL, 2006).

Este trabalho teve como objetivo realizar uma análise de custo-efetividade e do impacto orçamentário de tratamentos indicados para adultos infectados com genótipo 1 do vírus da hepatite $\mathrm{C}$, comparando-se o não-tratamento com terapias combinadas de alfapeguinterferon- $2 \mathrm{a}$ e $2 \mathrm{~b}$ associados a ribavirina.

\section{Métodos}

Um modelo de Markov de estados de transição da evolução da hepatite C para o genótipo 1 foi desenvolvido para se estimar os custos e benefícios anuais a partir da perspectiva do SUS. O modelo segue uma coorte hipotética de 1.000 indivíduos com infecção crônica por VHC, numa variedade de diferentes cenários de tratamento. Os estados de Markov incluem estágios histológicos definidos em termos dos critérios METAVIR (DHUMEAUX; MARCELLIN; LEREBOURS, 2003) e complicações da infecção no longo prazo, definidas como: cirrose compensada, cirrose descompensada e hepatocarcinoma primário, encefalopatia, hemorragia por varizes esofágicas, ascite e transplante hepático. Seu objetivo foi prever o progresso da história natural da doença, os estados de saúde pelos quais a coorte se distribui, quanto tempo os pacientes ficam em cada estado e os custos do SUS para tratar os pacientes em cada um desses estados de saúde, durante o período de 48 semanas. A comparação foi entre a terapia dual com alfapeguinterferon 2 a versus alfapeguinterferon $2 \mathrm{~b}$, ambos associados à ribavirina. 
As probabilidades de transição obtidas determinaram os movimentos dos pacientes através dos diferentes estados de saúde. A cada ciclo, os pacientes podem permanecer em seu estado de saúde ou passar a outro estado segundo as probabilidades de transição. Existe ainda a possibilidade de pacientes com cirrose descompensada virem a receber um transplante hepático, bem como a falecer por outras causas não relacionadas à infecção pelo VHC. Por fim, em relação aos tratamentos, o modelo contemplou as probabilidades de suspensão dos tratamentos e de ajustes de dose.

As estimativas relativas à história natural da doença foram confrontadas e eventualmente corrigidas, para torná-las mais representativas da realidade brasileira. Nesse sentido, foram obtidas estatísticas nacionais procedentes de estudos específicos (prevalência da infecção pelo VHC na população geral e em subpopulaçôes específicas), bem como de bancos de dados como o Sistema Informação de Mortalidade, Sistema de Informação sobre Agravos de Notificação Compulsória. Estimativas de eficácia dos tratamentos foram retiradas do trabalho desenvolvido por Malone et al. (2005).

Tomando por base as evidências disponíveis na literatura, de forte ligação entre a evolução histológica e a presença do vírus, o ponto final de interesse utilizado no estudo proposto foram resultados negativos do HCV-RNA medidos ao final do tratamento e seis meses após o seu término (RVS). No tratamento com interferon peguilado, a ausência de resultados negativos do HCV-RNA após 12 semanas do início de tratamento foi considerada como critério de exclusão da continuidade do uso do medicamento, conforme preconizado pela Portaria $\mathrm{n}^{\circ}$ 34/2007 (BRASIL, 2007).

Por fim, alguns dos pressupostos relativos ao tratamento da infecção foram assumidos e incluem: (a) a infecção crônica pelo VHC pode ter resolução, como resultado do tratamento, que se expressaria por HCV-RNA quantitativo negativo; (b) pacientes com resposta sustentada ao tratamento não experimentam progressão histológica subsequente da fibrose; e (c) pacientes que não apresentam resposta sustentada ao tratamento não são objeto de tratamento posterior.

O estudo analisou as medidas de eficácia/efetividade: 1) resposta viral sustentada; 2) anos de vida ganhos; e 3 ) anos de vida ajustado por qualidade. 
Tabela 1 - Efetividade do tratamento da HVC com alfapeguinterferon $2 \mathrm{a}+$ ribavirina e alfapeguinterferon $2 \mathrm{~b}+$ ribavirina

\begin{tabular}{l|c|c}
\hline & $\begin{array}{c}\text { Alfapeguinterferon } \\
\mathbf{2 a}+\text { ribavirina }\end{array}$ & $\begin{array}{c}\text { Alfapeguinterferon } \\
\text { 2b + ribavirina }\end{array}$ \\
\hline Resposta viral sustentada (SVR) & 0,49 & 0,48 \\
\hline Resposta viral precoce (EVR) & 0,81 & 0,76 \\
\hline Probabilidade de se atingir SVR & 0,6049 & 0,6315 \\
\hline
\end{tabular}

Fonte: Malone et al. (2005).

As probabilidades de transição estão relacionadas na tabela abaixo.

Tabela 2 Probabilidades de transição entre os diferentes estados da evolução da Hepatite C

\begin{tabular}{l|c}
\hline \multicolumn{1}{c|}{ Estado } & Probabilidade de Transição \\
\hline cirrose para ascite & 0,025 \\
\hline ascite para ascite refratário & 0,067 \\
\hline hepatite crônica para cirrose compensada & 0,073 \\
\hline cirrose compensada para Encefalopatia Hepática & 0,004 \\
\hline hepatite crônica para hepatocarcinoma & 0,001 \\
\hline cirrose para hepatocarcinoma & 0,015 \\
\hline cirrose compensada para hemorragia gastrointestinal & 0,011 \\
\hline morte ascite & 0,11 \\
\hline morte ascite refratário & 0,33 \\
\hline morte encefalopatia hepática no primeiro ano & 0,68 \\
\hline morte encefalopatia hepática anos subsequentes & 0,4 \\
\hline morte hepatocarcinoma & 0,86 \\
\hline morte hemorragia gastrointestinal primeiro ano & 0,4 \\
\hline morte hemorragia gastrointestinal anos subsequente & 0,13 \\
\hline hepatite leve para moderada & 0,041 \\
\hline morte transplante primeiro ano & 0,21 \\
\hline morte transplante anos subsequente & 0,057 \\
\hline inte: Lo & $W 000), B e n c t$ \\
\hline
\end{tabular}

Fonte: Loubiere (2003), Salomon (2003), Siebert (2003), Singer (2001), Wong (2000), Bennett (1997) e Kim (1997). 
Os preços dos peguinterferons foram obtidos através de dados disponíveis de licitações, aplicando-se os preços médios encontrados no Portal de Compras do Governo Federal (BRASIL, 2009d). Para os demais medicamentos utilizados, foi empregado o Banco de Preços da Câmara de Medicamentos/CMED, adotandose os valores com o menor encargo de ICMS, a fim de que esses refletissem melhor a realidade do pagador público, quando os dados de licitações não estivessem disponíveis (BRASIL, 2009c). Na ausência de informaçōes nesses bancos, se recorreu também ao mercado para obtenção dos preços dos medicamentos com venda liberada ao comércio.

Considerando que a perspectiva de análise utilizada no estudo foi a do SUS, foram levantados aqueles custos diretamente envolvidos no cuidado prestado pelo sistema de saúde, gerados pela utilização de recursos no tratamento da hepatite $\mathrm{C}$, incluindo tratamento hospitalar, ambulatorial, serviços médicos e de enfermagem, exames laboratoriais e medicamentos, entre outros. Como medidas de valoração dos custos mencionados, foram usados os valores de reembolso pagos pelo SUS para os diferentes itens, encontrados no Sistema de Gerenciamento da Tabela de Procedimentos, Medicamentos e OPM do SUS (SIGTAP) (BRASIL, 2009b). Estes se basearam nas suas frequências anuais, tomando por base as prescrições de utilização de recursos presentes no Protocolo para Tratamento da Hepatite C do Ministério da Saúde (BRASIL, 2007), bem como, quando necessário, levantamento junto a médicos especialistas (em especial, hepatologistas, infectologistas, intensivistas e oncologistas) que participam do tratamento da hepatite $\mathrm{C}$ no setor público, para estimativas mais precisas das quantidades de recursos absorvidas, levando em conta, entre outros, o tempo médio de internação para as várias complicações (ascite, encefalopatia, sangramento pelas varizes, câncer hepático, etc.). Dada a opção por esta ótica, não foram considerados outros itens de custo que seriam relevantes sob perspectivas diferentes, como por exemplo, a da sociedade, onde seria necessário incluir também estimativas de custos relativas ao absenteísmo ao trabalho, perda de produtividade, transporte e deslocamento dos pacientes e seus acompanhantes, etc. A tabela a seguir relaciona o custo total de cada etapa. 


\begin{tabular}{|c|c|}
\hline Procedimento & Custo total (R\$) \\
\hline Exames Iniciais & 624,62 \\
\hline $\begin{array}{l}\text { Exames Monitoramento } \\
\text { Peguinterferon } 48 \text { semanas } \\
\text { Peguinterferon } 12 \text { semanas (exclusão do tratamento) } \\
\text { Follow up Interferon/Peguinterferon } 48 \text { semanas }\end{array}$ & $\begin{array}{c}595,55 \\
268,28 \\
116\end{array}$ \\
\hline $\begin{array}{l}\text { Medicação } \\
\text { Peguinterferon 2a 180mcg (48 semanas) } \\
\text { Peguinterferon 2b } 120 \mathrm{mcg} \text { (48 semanas) } \\
\text { Peguinterferon 2a 180mcg (12 semanas) } \\
\text { Peguinterferon 2b 120mcg (12 semanas) }\end{array}$ & $\begin{array}{l}47.052,00 \\
61.341,12 \\
6.972,00 \\
15.524,28 \\
\end{array}$ \\
\hline $\begin{array}{l}\text { Cirrose Compensada } \\
\text { Diagnóstico } \\
\text { Acompanhamento }\end{array}$ & $\begin{array}{c}72,78 \\
188,16\end{array}$ \\
\hline $\begin{array}{l}\text { Cirrose com Varizes } \\
\text { Diagnóstico } \\
\text { Acompanhamento }\end{array}$ & $\begin{array}{l}143,93 \\
153,95 \\
\end{array}$ \\
\hline $\begin{array}{l}\text { Cirrose com Ascite } \\
\text { Diagnóstico do líquido ascítico } \\
\text { Tratamento ascite } \\
\text { Acompanhamento }\end{array}$ & $\begin{array}{c}41,85 \\
244,54 \\
374,76 \\
\end{array}$ \\
\hline $\begin{array}{l}\text { Encefalopatia Hepática } \\
\text { Diagnóstico } \\
\text { Tratamento }\end{array}$ & $\begin{array}{c}93,04 \\
1.009,04\end{array}$ \\
\hline $\begin{array}{l}\text { Varizes Gastresofágicas } \\
\text { Diagnóstico } \\
\text { Tratamento } \\
\text { Acompanhamento } \\
\end{array}$ & $\begin{array}{c}68,16 \\
7.698,90 \\
264,48 \\
\end{array}$ \\
\hline $\begin{array}{l}\text { Hepatocarcinoma } \\
\text { Diagnóstico } \\
\text { Tratamento Quimioterápico }\end{array}$ & $\begin{array}{c}197,89 \\
3.007,76\end{array}$ \\
\hline $\begin{array}{l}\text { Transplante Hepático } \\
\text { Transplante } \\
\text { Acompanhamento Pós Transplante }\end{array}$ & $\begin{array}{c}60.939,37 \\
4.594,41\end{array}$ \\
\hline
\end{tabular}

Fonte: elaboração própria. 
No estudo, não foi previsto o desenvolvimento de uma medida nacional para a qualidade de vida. Para superar esta ausência de informaçôes, solicitouse que dois hepatologistas pudessem avaliar as medidas de utilidade usadas em modelos internacionais. $\mathrm{O}$ valor final dessa análise encontra-se na tabela abaixo. Os valores foram objetos também da análise de sensibilidade, supondo-se: a) que os valores fossem iguais a um, isto é, medindo apenas os anos de vida; e b) o pior cenário imaginado.

Tabela 4 - Utilidades entre os diferentes estados da evolução da Hepatite C

\begin{tabular}{l|c|c}
\hline \multicolumn{1}{c|}{ Estados de Saúde } & Utilidades & Intervalo \\
\hline ascite & 0,65 & $0,35-1,0$ \\
\hline ascite refratário & 0,65 & $0,35-1,0$ \\
\hline cirrose & 0,82 & $0,46-1,0$ \\
\hline hepatoCarcinoma & 0,55 & $0,15-1,0$ \\
\hline gastrointestinal & 0,53 & $0,19-1,0$ \\
\hline varizes hemorrágicas & 0,55 & $0,23-1,0$ \\
\hline hepatite moderada & 0,98 & $0,92-1,0$ \\
\hline hepatite ao fim do tratameto & 0,92 & $0,72-1,0$ \\
\hline pós-transplante hepático & 0,86 & $0,66-1,0$ \\
\hline
\end{tabular}

Fonte: elaboração própria.

\section{Resultados}

\section{Análise de custo-efetividade}

O modelo de Markov desenvolvido projetou a evolução da hepatite C de pacientes com genótipo 1 para os vários estados do desenvolvimento da doença, em uma coorte de 1.000 pacientes, por um período de 30 anos (tabela 5). As terapias combinadas da ribavirina com o alfapeguinterferon $2 \mathrm{a}$ ou com o alfapeguinterferon $2 \mathrm{~b}$ apresentam efetividades estatisticamente idênticas, quando avaliadas a evolução da doença em 30 anos. 
Hepatite C crônica com genótipo 1

\begin{tabular}{|c|c|c|c|}
\hline Estados da doença & $\begin{array}{l}\text { História } \\
\text { Natural }\end{array}$ & $\begin{array}{c}\text { Alfapeguinterferon } \\
2 \mathrm{a}+\text { ribavirina } \\
\end{array}$ & $\begin{array}{c}\text { Alfapeguinterferon } \\
\text { 2b + ribavirina }\end{array}$ \\
\hline Hepatite Moderada & 0 & 0 & 0 \\
\hline Resposta ao fim do tratamento & 0 & 0 & 0 \\
\hline Curado & 0 & 392 & 384 \\
\hline $\begin{array}{l}\text { Hepatite moderada pós } \\
\text { tratamento }\end{array}$ & 86 & 46 & 47 \\
\hline Cirrose & 265 & 138 & 140 \\
\hline HepatoCarcinoma & 5 & 3 & 3 \\
\hline Ascite & 39 & 20 & 21 \\
\hline Ascite refratário & 7 & 4 & 4 \\
\hline Encefalopatia hepática & 1 & 1 & 1 \\
\hline Encefalopatia hepática>lano & 1 & 0 & 0 \\
\hline Hemorragia gastrointestinal & 3 & 2 & 2 \\
\hline $\begin{array}{l}\text { Hemorragia } \\
\text { gastrointestinal>1ano }\end{array}$ & 13 & 7 & 7 \\
\hline Transplante & 1 & 1 & 1 \\
\hline Transplante $>1$ ano & 11 & 6 & 6 \\
\hline Morte_Hepatocarcinoma & 142 & 71 & 72 \\
\hline Morte por Outras causas & 153 & 177 & 177 \\
\hline Morte Doença Hepática & 272 & 134 & 138 \\
\hline
\end{tabular}

Fonte: elaboração própria.

A tabela a seguir mostra o impacto do tratamento nos anos de vida e nos anos de vida ajustados por qualidade de vida para um período de 30 anos. Os anos de vida ganhos por qualidade de vida em relação à evolução da doença sem tratamento foi de 1,67 e 1,63, respectivamente, para a terapia combinada com alfapeguinterferon $2 \mathrm{a}$ e alfa $2 \mathrm{~b}$, aplicando-se $5 \%$ de desconto. Estaticamente não existe diferença entre o uso dos dois tipos de alfapeguinterferon. 
Tabela 6 - Anos de vida ganhos de acordo com as estratégias de tratamento

\begin{tabular}{c|c|c|c}
\hline Efetividade & $\begin{array}{r}\text { História } \\
\text { Natural }\end{array}$ & $\begin{array}{c}\text { Alfapeguinterferon 2a } \\
\text { mais Ribavirina }\end{array}$ & $\begin{array}{c}\text { Alfapeguinterferon } \\
\text { 2b mais Ribavirina }\end{array}$ \\
\hline QUALY (5\% desconto) & 12,19 & 13,86 & 13,82 \\
\hline QUALY (sem desconto) & 20,70 & 24,74 & 24,64 \\
\hline QUALY (10\% desconto) & 8,37 & 9,20 & 9,18 \\
\hline
\end{tabular}

Fonte: elaboração própria.

O resultado da análise de custo efetividade pode ser vista na tabela 7 , a seguir. A estratégia de tratamento com alfapeguinterferon $2 \mathrm{a}$ mais ribavirina se revelou mais custo-efetiva, dominando a alternativa do tratamento com alfapeguinterferon $2 \mathrm{~b}$ mais ribavirina. Embora não haja diferenças significativas de efetividade entre os dois tipos de interferon peguilado, em média apenas 0,04 em 30 anos, a diferença de preço entre os dois medicamentos faz com que a alternativa do uso do alfapeguinterferon 2 a mais ribavirina seja mais eficiente.

Tabela 7 - Custo-efetividade das estratégias de tratamento da Hepatite C, para uma coorte de 1.000 pacientes por 30 anos

\begin{tabular}{|c|c|c|c|c|c|c|}
\hline Estratégia & Custo (R\$) & Custo Incr (R\$) & Efetividade (Qualys) & Efetiv Incr & $\mathrm{C} / \mathrm{E}$ & (ICER) \\
\hline História natural & $6.621,29$ & & 12,19 & & 543,24 & \\
\hline PegInter alfa 2a + Ribavirina & $44.744,72$ & $38.123,44$ & 13,86 & 1,68 & $3.227,39$ & $22.753,60$ \\
\hline Peginter alfa $2 \mathrm{~b}+$ Ribavirina & $54.574,48$ & $9.829,76$ & 13,82 & 0,04 & $3.948,14$ & (Dominado) \\
\hline \multicolumn{7}{|c|}{ Todas as opçôes relacionadas à História Natural } \\
\hline História natural & $6.621,29$ & & 12,19 & & 543,24 & \\
\hline PegInter alfa 2a + Ribavirina & $44.744,72$ & $38.123,44$ & 13,86 & 1,68 & $3.227,39$ & $22.753,60$ \\
\hline Peginter alfa $2 b+$ Ribavirina & $54.574,48$ & $47.953,20$ & 13,82 & 1,63 & $3.948,14$ & $29.342,48$ \\
\hline
\end{tabular}

Fonte: Elaboração própria. Custos em reais e efetividade em QALYS.

\section{Análise de sensibilidade}

Como os resultados de todos os modelos econômicos estão sujeitos a incertezas e imprecisóes, é essencial testar a extensão em que os resultados diferem de acordo com variações nas estimativas e pressupostos. Assim, foi realizada uma análise de sensibilidade para as estimativas de custo-efetividade correspondentes ao pior e ao melhor cenário para os seguintes elementos: (a) as probabilidades de transição entre os vários estados da hepatite $\mathrm{C}$ crônica, 
em especial as taxas de transição da hepatite crônica leve para moderada e da hepatite crônica moderada para cirrose; (b) as estimativas de efetividade atribuídas a cada um dos tratamentos selecionados, incluindo eventuais diferenciais entre as apresentações comerciais de alfapeguinterferon $2 \mathrm{a}$ e $2 \mathrm{~b}$; (c) a taxa de desconto, de acordo com o proposto pelas Diretrizes Metodológicas de Avaliação Econômica do Ministério da Saúde (BRASIL, 2009e); (d) os anos de vida ajustados por qualidade e (e) os preços dos medicamentos.

A tabela seguinte mostra o resultado da análise univariada da taxa de desconto. Os resultados alcançados mostram que essa variável não tem influência no produto final da avaliação do custo efetividade.

Tabela 8 - Análise de sensibilidade da variação da taxa de desconto

\begin{tabular}{|c|c|c|c|c|c|c|c|}
\hline $\begin{array}{l}\text { Taxa de } \\
\text { desconto }\end{array}$ & Estratégia & Custo (R\$) & Incr Custo & Efet & Incr Efet & $\mathrm{C} / \mathrm{E}(\mathrm{R} \$)$ & Incr C/E (ICER) (R\$) \\
\hline \multirow[t]{3}{*}{0} & História natural & $14.237,82$ & & 20,7 & & 687,98 & \\
\hline & PegInter alfa $2 a+$ Ribavirina & $48.594,74$ & $\mathrm{R} \$ 34.356,92$ & 24,74 & 4,04 & $1.964,31$ & $\mathrm{R} \$ 8.496,55$ \\
\hline & Peginter alfa $2 \mathrm{~b}+$ Ribavirina & $58.505,50$ & & 24,64 & & $2.374,28$ & (Dominado) \\
\hline \multirow[t]{3}{*}{0,025} & História natural & $9.494,65$ & & 15,52 & & 611,69 & \\
\hline & PegInter alfa 2a + Ribavirina & $46.194,09$ & $36.699,44$ & 18,06 & 2,54 & $2.557,18$ & $14.433,68$ \\
\hline & Peginter alfa $2 \mathrm{~b}+$ Ribavirina & $56.054,82$ & & 18 & & $3.113,71$ & (Dominado) \\
\hline \multirow[t]{3}{*}{0,05} & História natural & $6.621,29$ & & 12,19 & & 543,24 & \\
\hline & PegInter alfa $2 a+$ Ribavirina & $44.744,72$ & $38.123,44$ & 13,86 & 1,68 & $3.227,39$ & $22.753,60$ \\
\hline & Peginter alfa $2 b+$ Ribavirina & $54.574,48$ & & 13,82 & & $3.948,14$ & (Dominado) \\
\hline \multirow[t]{3}{*}{0,075} & História natural & $4.810,76$ & & 9,95 & & 483,71 & \\
\hline & PegInter alfa $2 \mathrm{a}+$ Ribavirina & $43.834,79$ & $39.024,03$ & 11,1 & 1,15 & $3.948,89$ & $33.789,82$ \\
\hline & Peginter alfa $2 b+$ Ribavirina & $53.644,58$ & & 11,07 & & $4.845,14$ & (Dominado) \\
\hline \multirow[t]{3}{*}{0,1} & História natural & $3.626,06$ & & 8,37 & & 432,98 & \\
\hline & PegInter alfa $2 \mathrm{a}+$ Ribavirina & $43.241,65$ & $39.615,59$ & 9,2 & 0,83 & $4.697,74$ & $47.717,86$ \\
\hline & Peginter alfa $2 b+$ Ribavirina & $53.038,04$ & & 9,18 & & $5.775,08$ & (Dominado) \\
\hline
\end{tabular}

Fonte: elaboração própria. Custos em reais e efetividade em QALYS.

Embora exista uma relação entre os custos e efetividade, mudanças nesta última não têm impacto significativo na primeira, o que significa que a diferença 
dos custos do tratamento permanecem relativamente estáveis vis à vis o aumento dos anos de vida ganhos por qualidade de vida.

A variação do preço de aquisição dos dois peguinterferons é uma variável fundamental. Como não existe uma diferença significativa de efetividade entre eles, o resultado da análise de custo efetividade vai depender fundamentalmente do custo de cada medicamento.

\section{Impacto orçamentário}

Uma estimativa do impacto orçamentário da utilização dos medicamentos no tratamento da hepatite $\mathrm{C}$ foi realizada, a fim de se avaliar o peso econômico de cada uma das alternativas. Para isto, projetou-se o número de doentes tratados a partir dos casos notificados em 2008, corrigidos pela taxa de crescimento populacional. ${ }^{1}$ A tabela a seguir estima a população a ser tratada para os próximos dez anos.

Tabela 9 - Estimativa dos novos casos de hepatite C que devem ser tratados no período 2008 a 2017 pelo SUS

\begin{tabular}{c|c|c}
\hline Ano & População $^{1}$ & Número de casos confirmados $^{2}$ \\
\hline 2008 & 189.612 .814 & 10.708 \\
\hline 2009 & 191.480 .630 & 10.813 \\
\hline 2010 & 193.252 .604 & 10.914 \\
\hline 2011 & 194.932 .685 & 11.008 \\
\hline 2012 & 196.526 .293 & 11.098 \\
\hline 2013 & 198.043 .320 & 11.184 \\
\hline 2014 & 199.492 .433 & 11.266 \\
\hline 2015 & 200.881 .685 & 11.344 \\
\hline 2016 & 202.219 .061 & 11.420 \\
\hline 2017 & 203.510 .422 & 11.493 \\
\hline
\end{tabular}

Fonte: ${ }^{1}$ IBGE (2009); ${ }^{2}$ elaboração própria.

Para estimativa do custo do tratamento com interferon peguilado mais ribavirina, considerou-se a necessidade da realização dos exames iniciais, dos custos do monitoramento e dos custos com o medicamento ${ }^{2}$ propriamente dito, conforme descriminado na tabela 10. As duas tabelas a seguir apresentam o custo utilizando o alfapeguinterferon $2 \mathrm{a}$ e $2 \mathrm{~b}$. 
Tabela 10 - Custo do tratamento com alfapeguinterferon 2 a mais ribavirina em pacientes com Hepatite C

\begin{tabular}{|c|c|c|c|c|c|c|}
\hline $\begin{array}{l}\text { custo exame } \\
\text { inicial (R\$) }\end{array}$ & $\begin{array}{c}\text { custo } \\
\text { Monitoramento } \\
12 \text { semanas }(\mathrm{R} \$)\end{array}$ & $\begin{array}{c}\text { custo } \\
\text { Monitoramento } \\
48 \text { semanas (R\$) }\end{array}$ & $\begin{array}{c}\text { custo do } \\
\text { medicamento } 12 \\
\text { semanas }(\mathrm{R} \$)\end{array}$ & $\begin{array}{c}\text { custo do } \\
\text { medicamento } 48 \\
\text { semanas }(\mathrm{R} \$)\end{array}$ & $\begin{array}{c}\text { Follow up } 24 \\
\text { semanas }\end{array}$ & Total \\
\hline 6.688 .431 & 735.422 & $5.160 .720,60$ & $23.938 .162,32$ & $408.226 .009,68$ & 1.006 .124 & 445.754 .869 \\
\hline 6.754 .016 & 742.633 & $5.211 .325,35$ & $24.172 .894,02$ & $412.228 .972,98$ & 1.015 .989 & 450.125 .831 \\
\hline 6.817 .103 & 749.570 & $5.260 .002,30$ & $24.398 .683,56$ & $416.079 .442,44$ & 1.025 .479 & 454.330 .281 \\
\hline 6.875 .817 & 756.026 & $5.305 .305,60$ & $24.608 .824,32$ & $419.663 .047,68$ & 1.034 .312 & 458.243 .332 \\
\hline 6.932 .033 & 762.207 & $5.348 .681,10$ & $24.810 .022,92$ & $423.094 .159,08$ & 1.042 .768 & 461.989 .871 \\
\hline 6.985 .750 & 768.114 & $5.390 .128,80$ & $25.002 .279,36$ & $426.372 .776,64$ & 1.050 .849 & 465.569 .897 \\
\hline 7.036 .969 & 773.745 & $5.429 .648,70$ & $25.185 .593,64$ & $429.498 .900,36$ & 1.058 .553 & 468.983 .410 \\
\hline 7.085 .689 & 779.102 & 5.467.240,80 & $25.359 .965,76$ & $432.472 .530,24$ & 1.065 .882 & 472.230 .410 \\
\hline 7.133 .160 & 784.322 & $5.503 .869,00$ & $25.529 .866,80$ & $435.369 .913,20$ & 1.073 .023 & 475.394 .154 \\
\hline 7.178 .758 & 789.336 & $5.539 .051,35$ & $25.693 .061,22$ & $438.152 .925,78$ & 1.079 .882 & 478.433 .015 \\
\hline
\end{tabular}

Fonte: elaboração própria.

Tabela 11 - Custo do tratamento com alfapeguinterferon $2 \mathrm{~b}$ mais ribavirina em pacientes com Hepatite C

\begin{tabular}{l|c|c|c|c|c|c}
\hline $\begin{array}{c}\text { custo exame } \\
\text { inicial (R\$) }\end{array}$ & $\begin{array}{c}\text { custo } \\
\text { Monitoramento } \\
\mathbf{1 2} \text { semanas (R\$) }\end{array}$ & $\begin{array}{c}\text { custo } \\
\text { Monitoramento } \\
\mathbf{4 8} \text { semanas (R\$) }\end{array}$ & $\begin{array}{c}\text { custo do } \\
\text { medicamento 12 } \\
\text { semanas (R\$) }\end{array}$ & $\begin{array}{c}\text { custo do } \\
\text { medicamento 48 } \\
\text { semanas (R\$) }\end{array}$ & $\begin{array}{c}\text { Follow up 24 } \\
\text { semanas }\end{array}$ & Total \\
\hline 6.688 .431 & 735.422 & $4.842 .157,60$ & $39.461 .121,60$ & $497.513 .382,72$ & 944.017 & 550.184 .532 \\
\hline 6.754 .016 & 742.633 & $4.889 .638,60$ & $39.848 .067,60$ & $502.391 .875,92$ & 953.274 & 555.579 .505 \\
\hline 6.817 .103 & 749.570 & $4.935 .310,80$ & $40.220 .272,80$ & $507.084 .521,76$ & 962.178 & 560.768 .957 \\
\hline 6.875 .817 & 756.026 & $4.977 .817,60$ & $40.566 .681,60$ & $511.451 .934,72$ & 970.465 & 565.598 .742 \\
\hline 6.932 .033 & 762.207 & $5.018 .515,60$ & $40.898 .349,60$ & $515.633 .500,32$ & 978.400 & 570.223 .005 \\
\hline 6.985 .750 & 768.114 & $5.057 .404,80$ & $41.215 .276,80$ & $519.629 .218,56$ & 985.981 & 574.641 .746 \\
\hline 7.036 .969 & 773.745 & $5.094 .485,20$ & $41.517 .463,20$ & $523.439 .089,44$ & 993.211 & 578.854 .962 \\
\hline 7.085 .689 & 779.102 & $5.129 .756,80$ & $41.804 .908,80$ & $527.063 .112,96$ & 1.000 .087 & 582.862 .657 \\
\hline 7.133 .160 & 784.322 & $5.164 .124,00$ & $42.084 .984,00$ & $530.594 .212,80$ & 1.006 .787 & 586.767 .590 \\
\hline 7.178 .758 & 789.336 & $5.197 .134,60$ & $42.354 .003,60$ & $533.985 .927,12$ & 1.013 .223 & 590.518 .382 \\
\hline
\end{tabular}

Fonte: elaboração própria.

A utilização da opção mais custo-efetiva encontrada na análise desenvolvida, isto é, a utilização do alfapeguinterferon $2 \mathrm{a}$ mais ribavirina para o período de 2008 a 2017, significa uma redução nos gastos de aproximadamente 19\%, na perspectiva de que todos os doentes fossem tratados utilizando-se os esquemas terapêuticos selecionados. 


\section{Discussão}

A análise aqui realizada buscou incorporar a comparação do uso da terapia combinada da ribavirina com o alfapeguinterferon $2 \mathrm{a}$ ou $2 \mathrm{~b}$, na perspectiva do SUS, em um modelo extensivo de Markov da história natural da evolução da hepatite $\mathrm{C}$. $\mathrm{O}$ resultado aqui encontrado foi que o alfapeguinterferon 2a apresenta uma melhor eficiência, com uma redução de $19 \%$ nos gastos vis à vis o uso do alfapeguinterferon $2 \mathrm{~b}$.

Como não foram encontradas diferenças significativas entre a efetividade dos dois alfapeguinterferons, essa conclusão se deve exclusivamente ao preço do medicamento incorporado no modelo. Isto significa que a decisão entre um ou outro medicamento depende do preço que está sendo praticado. A análise de sensibilidade mostrou que a utilização do preço de um no outro medicamento implica mudança das conclusões alcançadas.

Uma análise da literatura internacional mostra alguns resultados contraditórios. Para Malone et al. (2005), a utilização de alfapeguinterferon 2b se revela mais eficiente, devido à maior probabilidade do valor preditivo da resposta viral sustentada. O estudo, no entanto, baseia-se em uma árvore de decisão, sem considerar a evolução da doença, nem utilizar como medida de efetividade a qualidade de vida dos doentes.

Yeh et al. (2007), por sua vez, utilizando um modelo de Markov para a evolução da história natural da hepatite $\mathrm{C}$ e com medidas de utilidade para avaliar o desfecho da evolução da doença, não encontraram resultado que desse preferência para um ou outro tipo interferon peguilado. Portanto, a conclusão do estudo foi que para o genótipo tipo 1 não existiriam recomendações privilegiando o uso de um ou outro medicamento. A principal divergência desse modelo para o aqui desenvolvido está na diferença de preço praticada nos EUA e no Brasil.

\section{Conclusão}

A hepatite crônica é assintomática na grande maioria dos casos, o que torna seu diagnóstico muito difícil. Infelizmente, as manifestações clínicas só aparecem quando o comprometimento hepático é irreversível. Na maioria das vezes, o diagnóstico precoce ocorre nos hemocentros, a partir da triagem em doadores de sangue ou na população que realiza exames de rotina regularmente. A 
progressão da doença e o diagnóstico precoce são fatores determinantes na vida de um indivíduo infectado.

Este estudo tem algumas limitaçôes. Primeiro, todas as probabilidades de transição foram retiradas de estudos internacionais e podem não refletir necessariamente as possibilidades de evolução da doença. Este fator, no entanto, tem um peso atenuado, na medida em que a análise de sensibilidade mostrou pequeno impacto no resultado final.

Segundo, o custo de aquisição do peguinterferon é, em grande medida, o responsável pelo resultado final alcançado. Como estes preços foram obtidos a partir de valores obtidos em algumas licitações, é possível que os valores não se mantenham. Em consequência, a recomendação do estudo é que a escolha mais custo-efetiva do uso do alfapeguinterferon $2 \mathrm{a}$ ou $2 \mathrm{~b}$ depende do preço que se pode obter na aquisição de cada um desses medicamentos.

\section{Referências}

BRASIL. Ministério da Saúde. Portaria n o 1.259, de 17 de junho de 2009. Estabelece processo de aquisição centralizada pelo Ministério da Saúde, para o medicamento Ribavirina do Componente de Medicamentos de Dispensação Excepcional. Diário Oficial [da] República Federativa do Brasil, Brasília, DF, n.o 114, p. 41/47, jun. 2009a, Seção 1.

BRASIL. Ministério da Saúde. Datasus. Sistema de Gerenciamento da Tabela de Procedimentos, Medicamentos e OPM. Disponível em: <http://sigtap.datasus.gov.br/ tabela-unificada/app/sec/inicio.jsp> Acesso em: 02 de fev. 2009b.

BRASIL. Ministério da Saúde. Câmara de Regulação do Mercado de Medicamentos. Disponível em: <http://www.anvisa.gov.br/monitora/cmed/index.htm> Acesso em: 08 abr. 2009c.

BRASIL. Ministério do Planejamento, Orçamento e Gestão. Portal de Compras do Governo Federal. Disponível em: < http://www.comprasnet.gov.br> Acesso em: 27 mai. 2009d.

BRASIL. Ministério da Saúde. Secretaria de Ciência, Tecnologia e Insumos Estratégicos. Diretrizes Metodológicas: Estudos de Avaliação Econômica de Tecnologias em Saúde. Brasília: Ministério da Saúde, 2009e. 150 p. (Série A. Normas e Manuais Técnicos).

BRASIL. Ministério da Saúde. Portaria No 34, de 28 de setembro de 2007. Dispõe Sobre Protocolo Clínico e Diretrizes Terapêuticas para Hepatite Viral C. Diário Oficial [da] República Federativa do Brasil, Brasília, DF, no 195, p. 60, out. 2007. Seção 1.

BRASIL. Ministério da Saúde. Portaria n o 3.227, de 20 de dezembro de 2006. Estabelece processo de aquisição centralizada pelo Ministério da Saúde para o medicamento 
Alfapeginterferon do Componente de Medicamentos de Dispensação Excepcional. Diário Oficial [da] República Federativa do Brasil, Brasília, DF, no 244, p. 76, dez. 2006. Seção 1. BRASIL. Ministério da Saúde. Portaria SAS/MS no 863, de 29 de novembro de 2006. Estabelece o protocolo clínico e diretrizes terapêuticas - Hepatite Viral Crônica C. Diário Oficial [da] República Federativa do Brasil, Brasília, DF, no 229, p. 178 nov. 2006, Seção 1. BRASIL. Ministério da Saúde. Portaria GM/MS n 639, de 16 de junho de 2000. Aprova o protocolo clínico e diretrizes terapêuticas - Hepatite viral crônica tipo C-Interferon + Ribavirina. Diário Oficial [da] República Federativa do Brasil, Brasília, DF, p. 33 jun. 2000, Seção 1.

DHUMEAUX, D.; MARCELLIN, P.; LEREBOURS, E. Treatment of hepatitis C. The 2002 French Consensus. Gut, v. 52, n. 12, p. 1784-1787, 2003.

FONSECA, J.C.F. Epidemiologia da infecção pelo vírus da hepatite $\mathrm{C}$ no Brasil. Relatório do grupo de Estudo da Sociedade Brasileira de Hepatologia. Gastroenterologia e Endoscopia Digestiva, São Paulo, v. 18, p. 3-8, 1999.

HNATYSZYN, H. J. Chronic hepatitis C and genotyping: the clinical significance of determining HCV genotypes. Antiviral Therapy, v. 10, n. 1, p.1-11, 2005.

LAVANCHY, D. Public health measures in the control of viral hepatitis: A World Health Organization perspective for the next millennium. Journal of Gastroenterology and Hepatology, Canberra, v. 17, n. 4, p. S452-S459, 2002.

MALONE, D. C.; TRAN, T. T.; POORDAD, F. F. Cost-Efficacy Analysis of Peginterferon alfa-2b plus Ribavirin Compared With Peginterferon alfa-2a plus Ribavirin for the Treatment of Chronic Hepatitis C. Journal of Managed Care Pharmacy, Plainsboro, v. 11, n. 8, p.687-694, 2005.

MURRAY, J.L.; LOPEZ, A. D. Mortality by cause for eight regions of the world: Global Burden of Disease Study. The Lancet, v. 349, n. 9061, p.1269-1276, 1997.

WORLD HEALTH ORGANIZATION. World Health Organization Fact Sheet $\mathrm{n}^{\mathrm{o}}$ 164/2000. Disponível em: <http://www.who.int/mediacentre/factsheets/fs164/en/index. html > Acesso em: 14 jun. 2009.

YEH,W.S et al. Peginterferon alfa-2a versus peginterferon alfa-2b as initial treatment of hepatitis $\mathrm{C}$ virus infection: a cost-utility analysis from the perspective of the Veterans Affairs Health Care System. Pharmacotherapy, v. 27, n. 6, p. 813-824, 2007.

\section{Notas}

${ }^{1}$ Projeção do número de habitantes no Brasil de 2008 até 2017. Disponível em: < http://www.ibge. gov.br/espanhol/presidencia/noticias/noticia_visualiza.php?id_noticia $=1028 \& i d \_p a g i n a=1>$. Acesso em: 27 de mar. 2009.

${ }^{2}$ Para estimativa do custo do medicamento, utilizou-se o preço encontrado para a aquisição do Alfapeguinterferon 2a $180 \mathrm{mcg}$, a opção mais barata. 


\section{Abstract}

\section{Alfa-pegylated interferons (2a and 2b) and ribavirin for treatment chronic hepatitis $C$, genotype 1: a cost-effectiveness analysis}

A cost-effectiveness and budget impact of treatments given to adults infected with genotype 1 hepatitis $C$ virus was performed by comparing the non-treatment with combined therapy alfapeguinterferon- $2 \mathrm{a}$ and $2 \mathrm{~b}$ and ribavirin. The Markov model developed engineered the development of hepatitis $C$ in a cohort of 1,000 patients for a period of 30 years for the various states of disease development. The combined therapy of ribavirin with alfapeguinterferon $2 \mathrm{a}$ or $2 \mathrm{~b}$ have statistically identical effectiveness when evaluated at 30 years of disease. As for the impact of treatment in life years and years of life adjusted for quality of life, the life years gained for quality of life in relation to the evolution of the disease without treatment were 1.67 and 1.63 , respectively, for combined therapy with alfapeguinterferon $2 \mathrm{a}$ and $2 \mathrm{~b}$, applying a $5 \%$ discount. The strategy of treatment with alfapeguinterferon 2 a plus ribavirin was more costeffective, dominating the other treatment. Although there are significant differences in effectiveness between the two types of alfapeguinterferon, the price difference between the two products makes the alternative of using alfapeguinterferon 2a plus ribavirin more effective. Concerning the estimated budget impact for the period 2008 to 2017, using alfapeguinterferon 2 a plus ribavirin results in a reduction in spending of about $19 \%$ if all patients were treated using the selected treatment regimens.

> Key words: cost-effectiveness evaluation; chronic hepatitis C; genotype 1, pegylated interferons, ribavirin. 